\title{
CONSIDERAÇÕES ESTATÍSTICAS RELATIVAS À OITO SÉRIES DE PRECIPITAÇÃO PLUVIAL DA SECRETARIA DE AGRICULTURA E ABASTECIMENTO DO ESTADO DE SÃO PAULO
}

\author{
GABRIEL CONSTANTINO BLAIN \\ Instituto Agronômico - IAC, Campinas - SP, Brasil \\ gabriel@iac.sp.gov.br
}

Recebido Agosto 2008 - Aceito Janeiro 2009

RESUMO

O correto entendimento das diversas sucessões dos tipos de tempo, que podem ser observadas em uma região, é uma das etapas fundamentais na redução do risco climático associado ao setor agrícola. O objetivo do trabalho foi analisar a variabilidade temporal dos dados mensais de precipitação pluvial de oito localidades do Estado de São Paulo. Investigações sobre possíveis tendências climáticas também foram realizadas. Por meio da análise de ondeletas, do teste da razão da máxima verossimilhança e do teste de Mann-Kendall, observa-se elevada variabilidade temporal dos valores de precipitação pluvial mensal nas oito regiões analisadas. $\mathrm{O}$ tratamento de tais séries como sendo estritamente estacionárias, ou a condução de análises apenas no domínio da freqüência acarretará em perda de informações sobre as forçantes moduladoras desse processo estocástico. Apesar dessa característica, não houve detecção de marcantes tendências de ordem climática no regime de precipitação pluvial. Sob o ponto de vista agrometeorológico, essa elevada variabilidade imprime, em um zoneamento, considerável grau de incerteza na determinação do potencial de atendimento hídrico às culturas. Tal incerteza deve ser considerada na determinação de áreas aptas, inaptas e marginais.

Palavras Chave: Séries não estacionárias, zoneamento agrícola, tendências climáticas

\begin{abstract}
STATISTICAL CONSIDERATIONS OF EIGHT MONTHLY PRECIPITATIONS SERIES OF THE STATE OF SÃO PAULO, BRAZIL.

The correct understanding of the weather variability is a fundamental step in reducing the agricultural climate risk. The aim of the study was to evaluate the temporal variability of monthly precipitation data from eight regions of the State of São Paulo, Brazil. Investigations related to possible climate trends were also held. Using the wavelet analysis, the likelihood ratio test, and the Mann-Kendall test, it was observed a very high temporal variability of the monthly precipitation data in the eight analyzed regions. The treatment of such series as strictly stationary, or the use of statistical models (such as Fourier spectral analysis), that only reveals what frequency (spectral) components exist in the precipitation signal, will lead to the loss of important information about the modulating forcing of the precipitation temporal variability. Despite the high temporal variability, no trends on the rainfall series were detected. In the sense of agrometeorological applications, the high temporal variability of the monthly precipitation signal should be considerate on the agricultural zoning model of the State of São Paulo, Brazil.
\end{abstract}

KeyWords: Non stationary series, climate trends, agricultural zoning 


\section{INTRODUÇÃO}

Em outubro de 1995 o Ministério da Agricultura, Pecuária e Abastecimento (MAPA) instituiu, como fase inicial de implantação do programa de zoneamento agrícola, o projeto de redução de riscos climáticos na agricultura (Filho et al., 2005). De acordo com Keller (1998) e Schubnell (1998), entre as diversas etapas desse projeto, a quantificação dos riscos decorrentes de elementos climáticos adversos (principalmente aos relativos à precipitação pluvial), foi objeto de uma abordagem probabilística para determinar as chances de sucesso das diversas culturas em cada região e época de plantio.

Sob esse aspecto, o conhecimento da variabilidade espaço-temporal de longas séries meteorológicas, além de auxiliar na indicação de períodos e áreas mais propícias ao plantio/semeadura de culturas, pode também trazer informações extremamente importantes sobre possíveis periodicidades, tendências ou descontinuidades climáticas, auxiliando o estudo das forçantes moduladoras das condições meteorológicas em uma região. A união (e natural inter-relação) entre esses dois aspectos, oriundos do conhecimento da variabilidade espaçotemporal de longas séries, é etapa fundamental na redução do risco climático associado ao setor agrícola.

Sob o ponto de vista de análises matemático-estatísticas, Blain et al. (2008), por meio da distribuição gama e do teste da razão da verossimilhança $\left(\Lambda^{*}\right)$, indicam que as séries mensais de precipitação pluvial da região de Campinas-SP (18902006), não podem ser consideradas estritamente estacionárias. Segundo esses autores, essa "não-estacionaridade" indica que investigações relacionadas à periodicidade de picos de variância, não devem ser conduzidas apenas no domínio da freqüência. Tais análises precisam também considerar o domínio do tempo, já que diferentes períodos (amostras) podem apresentar picos de variância com distintas freqüências. Nesse sentido, Kayano e Blain (2007) indicam, para a região de Campinas-SP, que a potência global da ondeleta da precipitação pluvial mostra picos não significativos em 0,$6 ; 3,5$ e 32 anos. No caso do período de 32 anos, existe apenas indicação de potências (ou variâncias) temporalmente localizadas entre os anos de 1890 a 1960. Os picos de 0,6 e 3,5 anos são resultantes de potências significativas localizadas em esparsos e curtos intervalos de tempo espalhados no período de 1890 a 2006. Com isso, a necessidade de considerar o domínio tempo-freqüência torna-se evidente.

Haylock et al. (2006) analisando tendências em valores diários de precipitação na América do Sul (1960 a 2000), afirmam que o padrão de alteração observado nos valores extremos desse elemento meteorológico é, de modo geral, o mesmo para os totais anuais. Neste trabalho são verificadas mudanças para condições mais úmidas ("changes to wetter conditions") na parte sul do Brasil. Em estudos similares, voltados para a temperatura do ar, Vincent et al. (2005) observou tendências de elevação ligadas principalmente à temperatura noturna. Segundo esses autores, as estações em que foram detectadas tendências significativas concentram-se, especialmente, nas costas leste e oeste da América do Sul. Alexander et al. (2006) analisando dados globais extremos de temperatura e precipitação, na escala diária, identificaram em $70 \%$ das regiões analisadas, significativa elevação nas temperaturas noturnas. Segundo esses autores, houve também detecção de elevação nos dados relativos à precipitação pluvial. Entretanto, essas últimas alterações apresentaram uma coerência espacial bastante inferior a observada nos dados de temperatura do ar.

Estudos sobre tendências climáticas adquirem grande importância ao ser considerado que na agricultura brasileira, o mais importante fator de risco é a precipitação pluvial, pois a seca e a chuva excessiva respondem pela maioria dos sinistros agrícolas (Göpfert et al., 1993). Nesse sentido, Blain et al. (2008) afirmam também que a grande variabilidade amostral dos parâmetros da distribuição gama (ou dos dados de precipitação pluvial mensal) acarreta em elevado grau de incerteza na caracterização climática do regime de precipitação mensal na região de Campinas-SP. Entretanto, segundo esses autores, apesar dessa grande variabilidade amostral, não há indicações conclusivas de tendências de ordem climática no regime de precipitação mensal observado nos últimos 116 anos nessa região. Marengo et al. (2007) observaram que, no sudeste do Brasil, o total anual de precipitação parece não ter sofrido alteração nos últimos 50 anos.

Dessa forma, a fim de aprofundar as observações acima citadas para a região de Campinas, bem como estendê-las às demais localidades do Estado, o objetivo do trabalho foi analisar a variabilidade temporal dos dados mensais de precipitação pluvial de oito localidades do Estado de São Paulo. Investigações sobre possíveis tendências climáticas, também foram realizadas. Espera-se que essas análises forneçam contribuições importantes ao estado da arte do conhecimento da variabilidade temporal desse elemento meteorológico, minimizando o risco climático associado ao agronegócio paulista e brasileiro.

\section{MATERIAL E MÉTODOS}

Foram utilizados dados de precipitação pluvial mensal de oito localidades do Estado de São Paulo (Figura 1), pertencentes à Secretaria de Agricultura e Abastecimento (SAA). Por serem destinadas à pesquisa $\mathrm{e}$ ao monitoramento agrometeorológico,a coleta dos dados que compõem as séries utilizadas é feita de forma padronizado, rigorosa e (devido à seu uso rotineiro) consistida. As séries não apresentam falhas.

As séries temporais mensais foram denotadas por $\left\{\mathrm{X}_{\mathrm{t}}, \mathrm{t}=\right.$ $1,2,3 \ldots ., \mathrm{N}\}$, em que $\mathrm{N}$ é o número de meses contido em cada 
série temporal $(\mathrm{N}=118$ para cada uma das 12 séries mensais de Campinas-SP, por exemplo). Considera-se também que $\mathrm{t}$ $€$ à $T$ que é, por sua vez, caracterizado como o conjunto dos inteiros positivos $Z=\{1,2,3 \ldots$.$\} . A fim de facilitar a comparação$ entres as diferentes formas e escalas das funções densidade de probabilidade nas diferentes localidades, o período homogêneo de 1951 a 2007 foi escolhido para ser base inicial do ajuste das séries de precipitação à distribuição gama incompleta. Essa escolha considerou as indicações da Organização Mundial de Meteorologia, quanto ao espaço de tempo mínimo exigido para conduzir uma caracterização climática e a menor série disponível (Pindorama-SP). Blain (2005) por meio dos testes qui-quadrado e kolmogorov-Smirnov afirma que a distribuição de freqüências dos valores $\mathrm{Xt}$, oriundos de cada série mensal de precipitação mensal, pode ser representada pela distribuição gama. A função densidade de probabilidade gama (FDPG) é descrita por:

$$
g(X)=\frac{X^{\alpha-1} * e^{-x / \beta}}{\beta^{\alpha} \Gamma(\alpha)} \quad \text { para } X>0
$$

Em que T( $\alpha$ ) é a função gama, $\alpha$ e $\beta$ são, respectivamente, parâmetros de forma e escala da distribuição. Esses parâmetros foram calculados pelo método da máxima verossimilhança como recomendado e descrito por Wilks (2006) e Blain et al. (2006). Segundo Ben-Gai et al. (1998), a FDPG assume diversas formas, de acordo com a variação de $\alpha$ : valores desse parâmetro inferiores a 1 resultam em uma distribuição fortemente assimétrica (forma exponencial) com $\mathrm{g}(\mathrm{X})$ tendendo ao infinito para $X$ tendendo a $0 . \operatorname{Em} \alpha=1$ a função intercepta o eixo vertical em $1 / \beta$ para $X=0 . O$ aumento da magnitude desse parâmetro diminui o grau de assimetria (desvio em relação à moda) da distribuição (a densidade de probabilidade desloca-se para a direita). Valores de $\alpha$ maiores que 1 resultam na FDPG com ponto máximo (moda) em $\beta^{*}(\alpha-1)$.

Para fins agrometeorológicos, tais como o zoneamento agrícola, seria conveniente que a estrutura de probabilidade, caracterizada na amostra dos anos de 1951 a 2007, permanecesse constante em demais períodos futuros. Em outras palavras, seria interessante que o processo $\mathrm{x}(\mathrm{t})$ desenvolvesse-se no tempo, de modo que a escolha de uma origem $t$ não fosse importante (processo estritamente estacionário). Nesse sentido, Nakken (1998) afirma que análise de ondeletas é um método importante para analisar tendências não-estacionárias em séries de precipitação e de escorrimento superficial (runoff), revelando mudanças abruptas, pontos de quebra e descontinuidades nos sinais desses elementos. Essa autora afirma também que tais

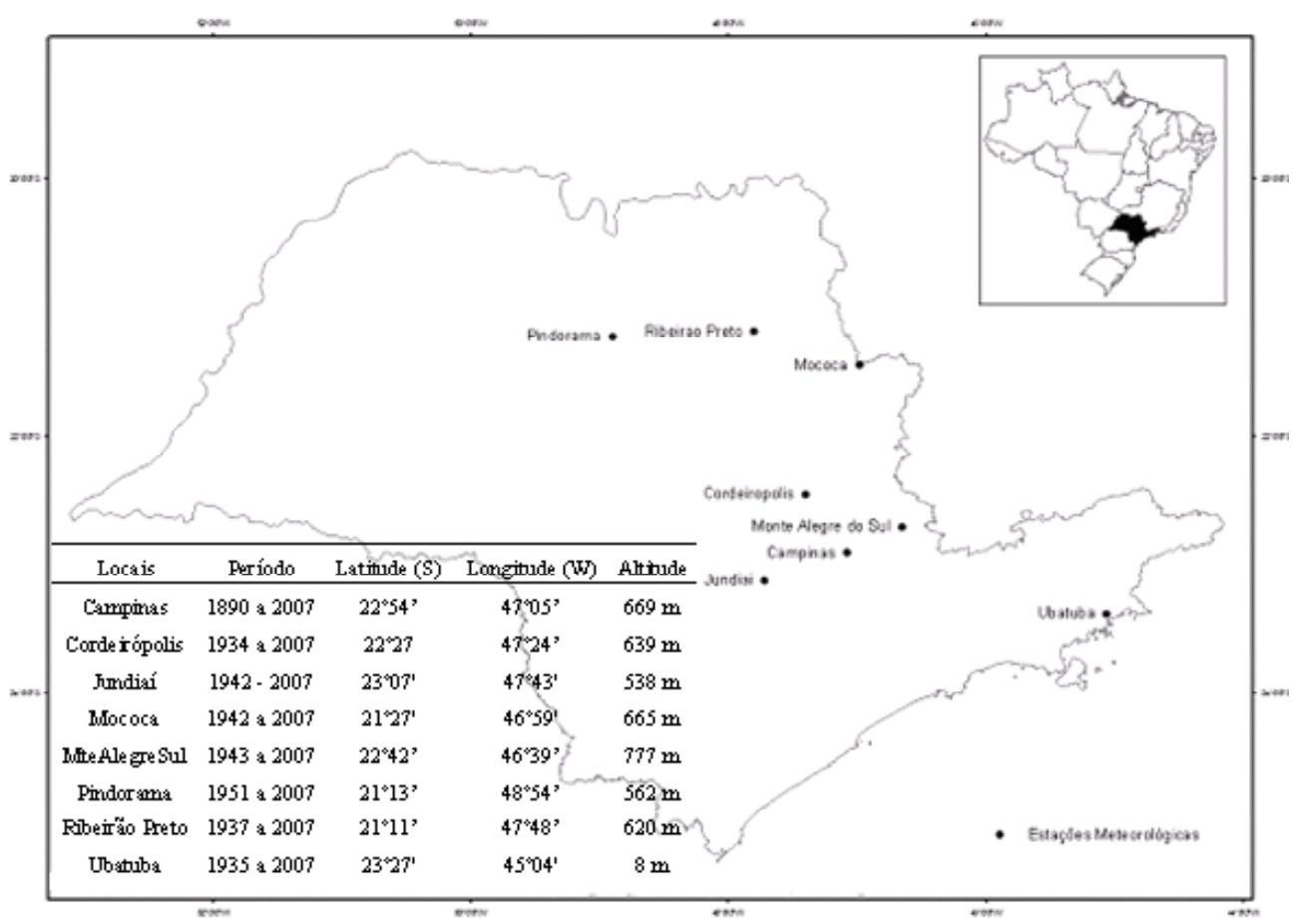

Figura 1 - Períodos disponíveis e coordenadas geográficas dos postos meteorológicos utilizados no estudo. Coordenadas geográficas cedidas pelo Instituto Agronômico (IAC). 
séries requerem, devido à natureza temporal de seus sinais, investigações baseadas no espaço tempo-freqüência a fim de revelar suas características espectrais como função do tempo. A análise de ondeleta foi aplicada para as séries (todo o período) de Cordeirópolis-SP, Jundiaí-SP, Mococa-SP, Monte Alegre do Sul-SP, Pindorama-SP, Ribeirão Preto-SP e Ubatuba-SP. Os resultados obtidos foram comparados aos de Kayano e Blain (2007) (realizados para o município de Campinas, utilizando a ondeleta - mãe de Mortet).

A ondeleta - mãe apropriada deve ter um padrão semelhante ao sinal em estudo (Nakken, 1998). Ainda segundo Nakken (1998) esse tipo de ondeleta - mãe, assim como as distribuições mensais de precipitação pluvial, apresenta pontos de máximos (picos superiores) e de mínimos (picos inferiores). Sob o mesmo aspecto, Torrence e Compo (1998) afirmam que tipos de ondeletas - mães como a de Morlet, são apropriadas para analisar a amplitude e a fase dos sinais sob avaliação sendo, com isso, indicadas para o estudo de séries temporais com características oscilatórias. Dessa forma, a função ondeleta - mãe utilizada foi a de Morlet. A climatologia das sete séries foi removida subtraindo-se as medianas mensais de cada valor Xt. Optou-se por trabalhar com a mediana devido a não-normalidade das distribuições utilizadas.

Admitindo-se a possibilidade de que as séries de precipitação do Estado de São Paulo não possam ser consideradas estritamente estacionárias, torna-se importante investigar a influência que a adoção de diferentes períodos amostrais pode ter na determinação do potencial de atendimento hídrico às culturas em uma região. O teste da razão da verossimilhança $\left(\Lambda^{*}\right)$ é indicado por Schickedanz e Krause (1969) e Wilks (2006) como sendo uma interessante alternativa para verificar se distintos períodos de uma série histórica de precipitação mensal podem ser considerados oriundos da "mesma distribuição gama". Assim, o teste compara a verossimilhança associada à uma hipótese de nulidade (Ho) à verossimilhança associada a uma hipótese alternativa HA. Ho considera que os distintos períodos sob teste pertencem à "mesma distribuição gama" e é rejeitada quando as verossimilhanças associadas à HA (definida como Ho não é verdadeira) são suficientemente elevadas para que as diferenças estatísticas entre os períodos sejam consideradas apenas variações amostrais. Uma interpretação relacionada é que a rejeição de Ho indica que a probabilidade de ocorrência associada a um dado valor de precipitação pluvial difere significativamente (ao nível escolhido) entre as amostras. Ho é rejeitado se o valor de $\Lambda^{*}$ for superior ao valor de um dado quantil (calda direita) da distribuição qui-quadrado. $\mathrm{O}$ quantil 0,9 (90\% de significância) dessa distribuição foi adotado como nível de significância do teste. Dessa forma, o valor crítico desse teste (considerando-se duas amostras) é 4,605. O teste $\Lambda^{*}$ é definido por Wilks (2006) da seguinte forma:

$$
\Lambda^{*}=2 \operatorname{Ln}[\Lambda(\mathrm{HA}) / \Lambda(\mathrm{Ho})]=2[\mathrm{~L}(\mathrm{HA})-\mathrm{L}(\mathrm{Ho})]
$$

Considerando-se duas amostras hipotéticas pertencentes a uma série dos meses de janeiro entre os anos de 1900 a 1999 o teste pode ser calculado por:

$\Lambda *=2\left\{\left[\sum_{i=1900}^{1999} L_{1}\left(\alpha_{1}, \beta_{1} ; x_{t}\right)+\sum_{i=1900}^{1949} L_{1}\left(\alpha_{2}, \beta_{2} ; x_{t}\right)\right]-\left[\sum_{i=1950}^{1999} L_{1}\left(\alpha_{3}, \beta_{3} ; x_{t}\right)\right\}\right.$

Em que:

$$
\mathrm{L}(\alpha, \beta ; \mathrm{xi})=(\alpha-1)^{*} \ln (\mathrm{xi} / \beta)-(\mathrm{xi} / \beta)-\ln (\beta)-\ln [\Gamma(\alpha)]
$$

Quanto maior a diferença entre os totais de precipitação observados nas amostras maior será a diferença entre os somatórios $\sum \mathrm{L}(\alpha, \beta ; \mathrm{xi})$ de cada amostra e, consequentemente, maior será o valor de $\Lambda^{*}$. É importante ressaltar que este método exige que os diferentes períodos amostrais sob análise possuam o mesmo número de dados. Dessa forma, o período homogêneo de 1951 a 2007 não permite a determinação de duas amostras com o mesmo número de dados (anos). Esse problema seria contornado adotando-se, para todas as localidades, o período de 1952 a 2007 (gerando duas amostras homogêneas de 28 anos). Em contra partida, à exceção da localidade de Pindorama, essa adoção resultaria na perda de grande número de dados nas demais localidades. Dessa forma, ainda que dificulte a comparação entre as localidades, optou-se por trabalhar com o maior número de dados possível (divididos em dois períodos amostrais) em cada região. As amostras mensais são: Cordeirópolis (1934/70 e 1971/07); Jundiaí (1942/74 e 1975/07); Mococa (1942/74 e 1975/07); Monte Alegre do Sul (1944/75 e 1976/07); Pindorama (1952/1979 e 1980/07); Ribeirão Preto (1938/1972 e 1973/2007); e Ubatuba (1936/71 e 1972/07). Nas amostras mensais em que observou-se $\Lambda *$ significativo, foram realizadas inferências sobre elevações ou quedas no regime de precipitação observado entre os dois períodos.

Apesar dessas inferências, a robustez do teste $\Lambda^{*}$ na detecção de quedas ou elevações em séries temporais de precipitação é limitada, pois a distribuição temporal (seqüência) dos valores de precipitação dentro de cada amostra mensal não é considerada. Esse tipo de análise pode ser realizado por meio do teste de Mann-Kendall (MK). O teste MK (Kendall e Stuart, 1967) é utilizado por Goosens e Berger (1986), Moraes et al. (1995), Back (2001) e Marengo et al. (2007), na detecção de tendências em séries temporais de precipitação pluvial. Moraes et al. (1995) e Back (2001) o descrevem por meio de uma série temporal de $\mathrm{X}_{\mathrm{i}}$ com $\mathrm{N}$ termos. $\mathrm{O}$ teste consiste na soma $\mathrm{t}_{\mathrm{n}}$ do número de termos mi da série, relativo ao valor $\mathrm{X}_{\mathrm{i}}$ cujos termos anteriores $(\mathrm{j}<\mathrm{i})$ são inferiores ao mesmo $\left(\mathrm{X}_{\mathrm{j}}<\mathrm{X}_{\mathrm{i}}\right)$, assim: 


$$
t n=\sum_{i=1}^{n} m i
$$

Devido ao fato das séries utilizadas nesse estudo serem longas, na ausência de tendências (hipótese de nulidade, Ho), tn apresentará distribuição normal com média $\left[\mathrm{E}\left(\mathrm{t}_{\mathrm{n}}\right)\right]$ e variância $\left[\operatorname{Var}\left(\mathrm{t}_{\mathrm{n}}\right)\right]$. O teste é calculado por:

$$
\begin{aligned}
& \mathrm{E}(\mathrm{tn})=\mathrm{N}(\mathrm{N}-1) / 4 \\
& \operatorname{Var}(\mathrm{tn})=\mathrm{N}(\mathrm{N}-1)(2 \mathrm{~N}+5) / 72 \\
& \mathrm{u}(\mathrm{t})=(\mathrm{tn}-\mathrm{E}(\mathrm{tn})) /(\operatorname{Var}(\mathrm{tn})) 1 / 2
\end{aligned}
$$

Adotando-se o nível de significância 5\%, utilizado por Moraes et al. (1995), Back (2001) e Marengo et al. (2007), Ho deve ser rejeitada sempre que o valor de $\mathrm{u}(\mathrm{t})$ estiver fora do intervalo de confiança $[-1,96 ;+1,96]$. Em outras palavras, se o valor de $\mathrm{u}(\mathrm{t})$ foi inferior à $-1,96$ há, de acordo com o teste MK, significativas tendências de queda nos valores da série sob investigação. Quando $\mathrm{u}(\mathrm{t})$ é superior a 1,96 há significativas tendências de elevação. O MK foi aplicado, separadamente, em cada série mensal de cada uma das oito localidades do estudo (8 localidades e 12 meses; totalizando 96 séries mensais; análogo ao teste $\Lambda^{*}$ ). A fim de facilitar o entendimento do teste MK, é apresentado o exemplo hipotético de uma série temporal $\mathrm{X}_{\mathrm{i}}$ com $\mathrm{N}=30$ termos contendo elevação em seus dados.

$\mathrm{Xi}=\{1 ; 2 ; 3 ; 4 ; 3 ; 3 ; 4 ; 5 ; 6 ; 1 ; 2 ; 3 ; 3 ; 4 ; 5 ; 35 ; 45 ; 35 ; 40 ; 35 ; 30 ; 40 ; 53 ; 50 ; 40$; $30 ; 32 ; 35 ; 35 ; 40\}$.

Os respectivos valores mi são (equação 5):

$\mathrm{mi}=\{0 ; 1 ; 2 ; 3 ; 2 ; 2 ; 5 ; 7 ; 8 ; 0 ; 2 ; 4 ; 4 ; 9 ; 12 ; 15 ; 16 ; 15 ; 17 ; 15 ; 15 ; 19 ; 22 ; 22 ; 19$; $15 ; 17 ; 18 ; 18 ; 13\}$.

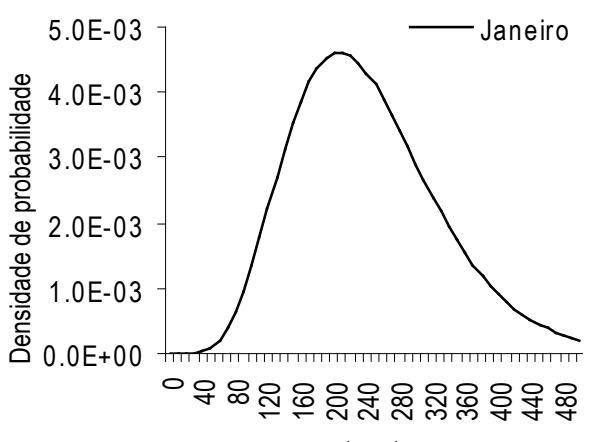

$(\mathrm{mm})$
No exemplo, $\mathrm{tn}=327 ; \mathrm{E}\left(\mathrm{t}_{\mathrm{n}}\right)=217,5 ; \operatorname{Var}(\mathrm{tn})=785,5 \mathrm{e} \mathrm{u}(\mathrm{t})$ $=3,907$ (significativo à 5\%). É importante observar que, quando há repetição de valores em $\mathrm{X}_{\mathrm{i}}$, o termo mi permanece constante. Dessa forma a tendência (significativa ou não significativa) que estava sendo observada antes da repetição dos valores é mantida. Para as séries mensais utilizadas, a repetição de valores ocorreu em menos de $1 \%$ dos casos.

\section{RESULTADOS E DISCUSSÃO}

Por meio da Tabela 1, à exceção da localidade de Ubatuba-SP, nota-se que o período entre os meses de novembro a março pode ser considerado como a época em que a estação chuvosa está plenamente estabelecida. Nesses meses, o alto valor do parâmetro $\alpha$ indica distribuições com formas próximas à normal, em que altos totais mensais de precipitação são climatologicamente esperados. Em contra partida, considerando que, segundo Wilhite (2000), a seca meteorológica ocorre quando há um desvio negativo nos valores de precipitação (chuva consideravelmente abaixo da normal) e que sua intensidade está, entre outros fatores, relacionada à magnitude desse desvio, essa época chuvosa é também sujeita à secas meteorológicas intensas. Sob o ponto de vista do calendário agrícola regional, esse período engloba fases de alta demanda hídrica por parte das culturas comerciais dessas sete localidades. Com isso, tais desvios negativos de precipitação, ainda que em curtos intervalos de tempo (veranicos) apresentam alto potencial de caracterizarem uma seca agrícola, que por sua vez ocorre quando o teor de umidade no solo é insuficiente para repor as perdas por evapotranspiração das culturas.

Em contrapartida a esse período chuvoso, nas localidades de Campinas, Monte Alegre do Sul, Cordeirópolis e Jundiaí, os meses de junho, julho e agosto são caracterizados por distribuições fortemente assimétricas, com valores do parâmetro $\alpha$ iguais ou inferiores a 1 . Nessa época, o valor zero de precipitação não representa, necessariamente, uma grande

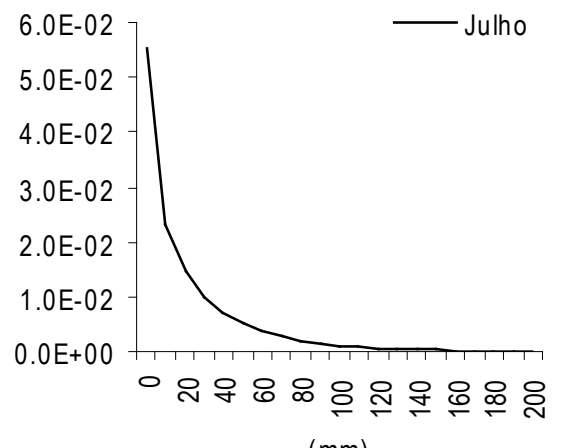

(mm)

Figura - 2 Forma gráfica da função densidade de probabilidade gama, ajustada aos dados mensais de precipitação pluvial (janeiro e julho) na localidade de Cordeirópolis-SP, 1934 a 2007. 
Tabela - 1 Valores dos parâmetros de forma $(\alpha)$ e escala $(\beta)$ da FDPG, média, desvio padrão (DP), coeficiente de variação (CV\%) e freqüência de meses sem chuva (Freq_0) nas localidades de Campinas, Cordeirópolis, Jundiaí, Mococa, Monte Alegre do Sul, Pindorama, Ribeirão Preto e Ubatuba pertencentes ao estado de São Paulo no período de 1951 a 2007.

\begin{tabular}{|c|c|c|c|c|c|c|c|c|c|c|c|c|}
\hline & \multicolumn{6}{|c|}{ Campinas-SP } & \multicolumn{6}{|c|}{ MonteAlegreSul-SP } \\
\hline Mês & Alfa & beta & Média & DP & $\mathrm{CV} \%$ & Freq_0 & Alfa & beta & Media & $\mathrm{DP}$ & CV\% & Freq \\
\hline Jan & 4,6 & 55,6 & 258 & 119,8 & 46,4 & 0,0 & 7,4 & 36,0 & 265 & 97,7 & 36,9 & 0,0 \\
\hline Fev & 3,5 & 56,2 & 199 & 105,7 & 53,2 & 0,0 & 3,3 & 62,6 & 209 & 114,4 & 54,7 & 0,0 \\
\hline Mar & 4,3 & 36,1 & 155 & 74,9 & 48,2 & 0,0 & 4,4 & 39,3 & 173 & 82,5 & & 0,0 \\
\hline $\mathrm{Abr}$ & 2,1 & 31,2 & 66 & 45,3 & 68,9 & 0,0 & 2,6 & 29,3 & 76 & 47,4 & 61,9 & 0,0 \\
\hline Maio & 1,2 & 57,1 & 68 & 62,5 & 91,3 & 0,0 & 1,4 & 51,5 & 71 & 60,6 & 85,0 & 0,0 \\
\hline Jun & 0,9 & 49,6 & 45 & 47,4 & 104,6 & 10,5 & 0,8 & 62,1 & 48 & 54,8 & 113,4 & 7,0 \\
\hline Jul & 0,7 & 48,3 & 34 & 40,8 & 118,3 & 14,0 & 0,7 & 49,5 & 37 & 42,9 & 115,5 & 8,8 \\
\hline Ago & 0,8 & 42,7 & 33 & 37,3 & 114,6 & 12,3 & 0,8 & 44,9 & 37 & 40,8 & 110,0 & 10,5 \\
\hline Set & 1,1 & 60,9 & 64 & 62,6 & 97,3 & 1,8 & 1,2 & 61,9 & 73 & 67,1 & 92,2 & 1,8 \\
\hline Out & 2,6 & 48,8 & 128 & 79,1 & 61,7 & 0,0 & 2,7 & 52,5 & 144 & 86,9 & 60,4 & 0,0 \\
\hline Nov & 4,1 & 34,8 & 141 & 70,1 & 49,7 & 0,0 & 4,5 & 36,3 & 164 & 77,2 & 47,1 & 0,0 \\
\hline \multirow[t]{2}{*}{ Dez } & 6,8 & & 212 & 81,2 & 38,3 & 0,0 & 8,6 & 27,3 & 234 & 80,1 & 34,1 & 0.0 \\
\hline & \multicolumn{6}{|c|}{ Cordeirópolis-SP } & \multicolumn{6}{|c|}{ Pindorama-SP } \\
\hline Mês & Alfa & beta & Média & $\mathrm{DP}$ & $\mathrm{CV} \%$ & Freq_0 & Alfa & beta & Média & $\mathrm{DP}$ & CV\% & req_o \\
\hline Jan & 5,8 & 43,4 & 251 & 104,3 & 41,6 & 0,0 & 5,1 & 51,3 & & 116,2 & 44,1 & 0,0 \\
\hline Fev & 3,2 & 61,9 & 196 & 110,2 & 56,1 & 0,0 & 3,7 & 58,3 & 216 & 112,1 & 52,0 & 0,0 \\
\hline Mar & 2,9 & 57,7 & 165 & 97,4 & 59,2 & 0,0 & 4,0 & 38,6 & 156 & 77,6 & 49,7 & 0,0 \\
\hline Abr & 1,9 & 35,9 & 67 & 49,1 & 73,2 & 0,0 & 1,4 & 48,5 & & 58,4 & 83,1 & 3,5 \\
\hline Maio & 1,2 & 55,0 & 66 & 60,2 & 91,3 & 0 , & 1,1 & 56,5 & 62 & 59,0 & 95,8 & 5,3 \\
\hline Jun & 0,8 & 50,4 & 42 & 45,8 & 110,0 & 10,5 & 0,7 & 45,2 & 31 & 37,7 & 120,0 & 17,5 \\
\hline Jul & 0,7 & 42,4 & 30 & 35,8 & 118,5 & 10,5 & 0,6 & 41,1 & 24 & 31,7 & 129,5 & 24,6 \\
\hline Ago & 0,7 & 46,0 & 30 & 37,2 & 123,6 & 19,3 & 0,6 & 39,6 & & 31,3 & 126,5 & 19,3 \\
\hline Set & 1,0 & 63,7 & 65 & 64,6 & 98,7 & & 0,9 & 65,0 & 5 & 61,5 & 105,7 & 7,0 \\
\hline Out & 3,3 & 38,3 & 128 & 70,0 & 54,8 & & 3,3 & 5,1 & 115 & 3,5 & & 0,0 \\
\hline Nov & 4,1 & 36,0 & 147 & 72,7 & 49,5 & & 3,7 & 36,5 & 136 & 70,4 & 51,9 & 0,0 \\
\hline \multirow[t]{2}{*}{ Dez } & 6,5 & 33,8 & 219 & 86,1 & 39,3 & & 3 & 1,0 & 216 & 94,1 & 43,6 & 0,0 \\
\hline & \multicolumn{6}{|c|}{ Jundiai-SP } & \multicolumn{6}{|c|}{ RibeirãoPreto-SP } \\
\hline Mês & Alfa & beta & Média & DP & $\mathrm{CV} \%$ & Freq_0 & Alfa & beta & Média & DP & CV\% & Freq_0 \\
\hline Jan & 4,8 & & & 115,5 & 45,6 & 0,0 & 5,1 & 55,0 & & 124,6 & 44,1 & 0,0 \\
\hline Fev & 3,7 & 49,5 & 184,7 & 95,6 & 51,8 & 0 , & 3,3 & 68,7 & 227 & 124,7 & 55 & 0,0 \\
\hline Mar & 4,2 & 37,2 & & 76,09 & 48,9 & & 3,7 & 44,5 & 166 & 85,8 & & 0,0 \\
\hline $\mathrm{Abr}$ & 1,7 & 40,7 & 69,42 & 53,14 & 76,5 & & 1,5 & 49,2 & & 61,1 & 80,7 & 0,0 \\
\hline Maio & 1,4 & 52,0 & & & & & 1,0 & 4,2 & & 63,6 & 0,9 & 1,8 \\
\hline & 0.9 & & 75 & & & & & & & 36.9 & & 17.5 \\
\hline Jul & 0,7 & 57,9 & & 50,09 & 115,7 & 14,0 & 0,6 & 0,9 & 2 & 31,2 & & 19,3 \\
\hline Ago & 0,9 & 42,9 & & 39,81 & 107,7 & & 0,6 & 38,1 & & 29,0 & 13 & 26,3 \\
\hline Set & 1,1 & 69,4 & 74,29 & 71,81 & 96,7 & & 0,9 & 61,3 & 55 & 58,3 & 105,2 & 3,5 \\
\hline & 3,9 & & & & 50 & & 3,2 & & & & & 0,0 \\
\hline Nov & 2.8 & 51,7 & 144,7 & 86.48 & 59,8 & & 4,1 & 42,7 & 176 & 86,8 & 49,2 & 0,0 \\
\hline & & 38,5 & 210,9 & 90,13 & 42,7 & & & & 274 & 111,1 & 40,5 & 0,0 \\
\hline \multicolumn{7}{|c|}{ Mococa-SP } & \multicolumn{6}{|c|}{ Ubatuba-SP } \\
\hline Mês & Alfa & beta & Média & $\mathrm{DP}$ & $\mathrm{CV} \%$ & $\overline{\text { Freq_0 }}$ & Alfa & beta & Média & $\mathrm{DP}$ & CV\% & Freq_0 \\
\hline $\mathrm{Ja}$ & 5,0 & & & 124 & 44,7 & & 3,1 & 114,9 & & 201,5 & 57,0 & 00 \\
\hline $\mathrm{Fev}$ & 3,3 & 64,7 & & 117,8 & 54,9 & & 1,9 & 174,6 & & & & \\
\hline Mar & 3.7 & & & & & & & & & & & \\
\hline Abr & 1,8 & 41,1 & & & 74,4 & & & & 2. & 121,9 & & 0,0 \\
\hline Maio & 1,3 & & & & & & & & & & & \\
\hline Jun & 0,7 & 45,9 & 31,49 & 38,03 & 120,8 & 17 & 1,8 & 45,3 & 81 & 60,7 & 74,7 & 0,0 \\
\hline Jul & 0,6 & 36,5 & & & & & & & & & & 0,0 \\
\hline Ago & 0,7 & & & 28,33 & 123 & & 2 , & & 90 & 54 & & 0 \\
\hline $\mathrm{S}$ & 1,0 & 63 , & 62,95 & 63 & 100,1 & & & 59,5 & 175 & 2,1 & 3 & 0,0 \\
\hline & 3 , & 42 & 135,3 & 76,04 & 56 & & 5,0 & 47,4 & 236 & 105,7 & 44 & 0,0 \\
\hline Nov & 5,0 & 35,0 & 175,5 & 78,37 & 44,7 & 0 & 4,1 & 65,6 & 269 & 132,8 & 49,4 & 0,0 \\
\hline Dez & 6,1 & 44,0 & 266,4 & 108,3 & 40,6 & 0,0 & 5,5 & 56,6 & 312 & 132,9 & 42,6 & 0,0 \\
\hline
\end{tabular}


anomalia (ou desvio) na sucessão climatologicamente esperada dos tipos de tempo que podem ser observados nesta época do ano. Assim, o estabelecimento (início) de secas meteorológicas durante esse período é, conceitualmente, improvável. Entretanto, se uma seca meteorológica, iniciada em outras épocas do ano, perdurar até esses meses (junho a agosto), a probabilidade de que essas distribuições mensais fortemente assimétricas forneçam suprimento hídrico suficiente para interromper esse tipo de seca é bastante baixa. Para as localidades de Pindorama e Mococa, essa época com distribuições “j” invertido ocorre entre os meses de junho a setembro, Para a localidade de Ribeirão Preto, essa época ocorre entre os meses de maio e setembro.

Nas localidades de Campinas, Monte Alegre do Sul, Cordeirópolis e Jundiaí, os meses de abril, maio, setembro e outubro podem ser considerados fases de transição entre as duas épocas acima citadas. Nas localidades de Pindorama e Mococa, essa fase de transição ocorre nos meses de abril, maio e outubro, ao passo que na região de Ribeirão Preto, esse período ocorre nos meses de abril e setembro. Na localidade litorânea de Ubatuba, não há grandes contrastes entre o regime de precipitação observado entre as estações do ano. Todos os meses apresentam distribuições com formas bastante próximas à normal. Nos meses de maio e junho observa-se os maiores graus de assimetria. A probabilidade de secas meteorológicas de longa duração é bastante baixa. Na Figura 2, é ilustrado as formas mensais da FDPG relativas um mês chuvoso (janeiro) e a um mês pertencente ao período seco (julho).

Analisando-se a Figura 3, observa-se que os picos de variância, mesmo quando apresentam picos significativos da potência global da ondeleta (GWP), ocorrem de forma esparsa ao longo das séries. Ressalta-se que Kayano e Blain (2007) observaram a mesma característica para a região de Campinas. Com isso, a natureza temporal dos picos de variância das séries mensais de precipitação pluvial das localidades analisadas, requer análises baseadas no espaço tempo-freqüência, a fim de revelar suas características espectrais como função do tempo.

A natureza esparsa dos picos de variância, observados na Figura 3 , indica que o processo $\mathrm{x}(\mathrm{t})$ desenvolve-se no tempo, de modo que a escolha de uma origem $t$ torna-se importante (indício de que o processo não é estritamente estacionário). Por meio da GWP verifica-se, para as regiões de Cordeirópolis, Jundiaí e Mococa, picos significativos de variância nas escala 2, 7 e 5 anos respectivamente. Para a região de Ubatuba-SP, há picos nas escalas 8 anos (que ocorrem por volta de 1950 a 1975), 16 e 20 anos (entre 1955 e 1975). Essa natureza esparsa também dificulta a correlação entre os picos de variância relativos às séries de precipitação pluvial e os picos de variância relativos ao sinal do El Niño/Oscilação Sul (ENOS). Nesse sentido, é interessante ressaltar Kayano e Blain (2007), que indicam que para as localidades do Estado de São Paulo, o sinal do ENOS na precipitação pluvial mensal não é claro. Na Tabela 2 são apresentados os resultados do teste $\Lambda^{*}$.

Após a detecção de $\Lambda^{*}$ significativos (Tabela 2), é interessante realizar inferências sobre quais períodos apresentaram queda (ou aumento) no regime de precipitação pluvial. A primeira tentativa de realizar tal análise foi baseada no fator $\mathrm{L}(\alpha, \beta ; \mathrm{xt})$ do teste $\Lambda^{*}$, que pode ser entendido como a contribuição de cada valor $\mathrm{Xt}$ ao somatório $\sum \mathrm{L}(\alpha, \beta ; \mathrm{xi})$. Entretanto, duas características impedem o uso de $\mathrm{L}(\alpha, \beta ; \mathrm{xt})$ na caracterização de queda (ou aumento) no regime de precipitação pluvial em uma amostra: a) valores extremos de Xt (ainda que possuam baixa freqüência de ocorrência dentro do período amostral) podem ter grande "peso" no valor final do somatório $\sum \mathrm{L}(\alpha, \beta ; \mathrm{xi})$. Em outras palavras, esse último fator, pode ser consideravelmente alterado por valores de precipitação contidos, por exemplo, em percentis superiores ao 0,90 ou inferiores ao 0,10 , das distribuições mensais. Com isso, as indicações de redução ou aumento no regime de precipitação pelo teste $\Lambda^{*}$, poderiam ser relativas a alterações amostrais em diversos percentis das distribuições consideradas, ou apenas relativas a alterações nos extremos da distribuição. b) a variação do fator $\mathrm{L}(\alpha, \beta ; \mathrm{xt})$ não é diretamente correlacionada à variação dos valores de precipitação pluvial. $\mathrm{O}$ valor, em módulo, de $\mathrm{L}(\alpha, \beta ; \mathrm{xt})$ diminui com o aumento do valor de precipitação pluvial, até atingir-se (aproximadamente) a mediana da amostra em análise. Após esse ponto, o valor, em módulo de $\mathrm{L}(\alpha, \beta ; \mathrm{xt})$ aumenta com a elevação dos valores de precipitação. A fim de contornar esse problema, os espaços amostrais de cada localidade foram organizados e comparados em forma de rol (ou rank; é a ordenação em forma crescente dos dados presentes em uma amostra. A posição 5 de um rol indica que está sendo considerado o quinto maior valor dessa amostra). Essa comparação ilustra a dificuldade presente em análises estatísticas voltadas à caracterização e, especialmente, detecção de alterações no regime de precipitação pluvial mensal no Estado de São Paulo.

Por meio dos rol de dados, observa-se que em alguns meses há diferenças, nos valores de precipitação, em todas as posições do rol de cada amostra, como por exemplo, nos meses de maio em Cordeirópolis; nos meses de maio e novembro em Jundiaí; nos meses de setembro em Mococa; nos meses de maio e setembro em Monte Alegre do Sul; nos meses de janeiro em Pindorama e nos meses de agosto em Ubatuba. Nesses meses, após a detecção de valores significativos do teste $\Lambda^{*}$, torna-se simples inferir, por meio dos valores do rol das amostras, em quais períodos houve queda/elevação no regime de precipitação pluvial. É importante ressaltar, que o termo elevação é aqui definido quando todos os valores dos rol relativos ao período mais recente, são superiores aos relativos ao período mais antigo da série em estudo. O termo queda é definido de forma oposta, 

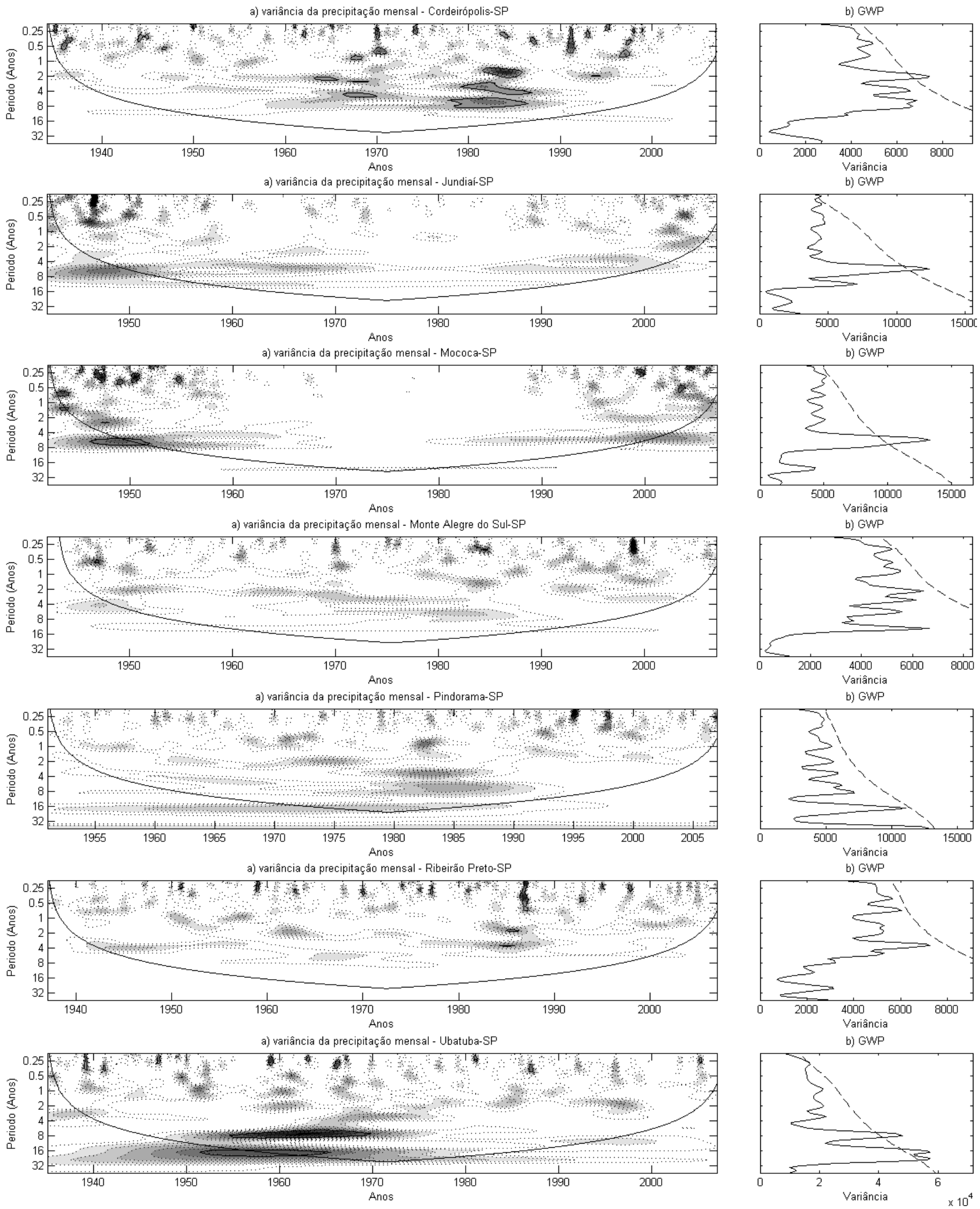

Figura 3 - Análises de ondeletas realizadas em sete localidades do Estado de São Paulo. Variância da precipitação mensal (a) temporalmente localizadas considerando-se diferentes períodos. Potência global da ondeleta (b; GWP) nos períodos analisados. 
Tabela 2 - Teste da razão da verossimilhança $\left(\Lambda^{*}\right)$ nas localidades de Cordeirópolis, Jundiaí, Mococa, Monte Alegre do Sul, Pindorama, Ribeirão Preto e Ubatuba, pertencentes ao Estado de São Paulo.

\begin{tabular}{|c|c|c|c|c|c|c|}
\hline & \multicolumn{2}{|c|}{ Cordeirópolis } & \multicolumn{4}{|c|}{ Jundiaí } \\
\hline & $1934 / 1970$ & $1971 / 2007$ & $\Lambda^{*}$ & $1942 / 1974$ & $1975 / 2007$ & $\Lambda^{*}$ \\
\hline Mês & $\sum \mathrm{L}(\alpha, \beta ; \mathrm{xi})$ & $\sum \mathrm{L}(\alpha, \beta ; \mathrm{xi})$ & & $\sum \mathrm{L}(\alpha, \beta ; \mathrm{xi})$ & $\sum \mathrm{L}(\alpha, \beta ; \mathrm{xi})$ & \\
\hline Jan & $-221,5$ & $-213,7$ & 1,85 & $-195,7$ & $-202,6$ & 1,50 \\
\hline Fev & $-221,0$ & $-218,6$ & 1,99 & $-192,7$ & $-192,8$ & 0,56 \\
\hline Mar & $-211,6$ & $-219,6$ & 3,32 & $-182,5$ & $-190,4$ & 2,94 \\
\hline $\mathrm{Abr}$ & $-184,7$ & $-191,7$ & 1,69 & $-155,6$ & $-166,2$ & 2,67 \\
\hline Maio & $-170,6$ & $-189,5$ & $7,92 *$ & $-149,2$ & $-177,1$ & $8,73^{*}$ \\
\hline Jun & $-155,4$ & $-163,3$ & 4,27 & $-162,7$ & $-156,0$ & 1,34 \\
\hline Jul & $-127,2$ & $-160,7$ & 1,72 & $-127,4$ & $-154,3$ & 0,31 \\
\hline Ago & $-143,6$ & $-128,5$ & 0,97 & $-126,9$ & $-140,4$ & 0,26 \\
\hline Set & $-188,1$ & $-182,4$ & $5,06^{*}$ & $-158,5$ & $-178,6$ & 4,06 \\
\hline Out & $-201,4$ & $-202,4$ & 2,00 & $-179,1$ & $-180,3$ & 0,50 \\
\hline Nov & $-203,3$ & $-209,1$ & 1,07 & $-185,0$ & $-187,2$ & $5,29^{*}$ \\
\hline \multirow[t]{3}{*}{ Dez } & $-221,4$ & $-213,0$ & 2.02 & $-196,2$ & $-189,3$ & 4,29 \\
\hline & & Mococa & & \multicolumn{3}{|c|}{ MteAlegreSul } \\
\hline & $1942 / 1974$ & $1975 / 2007$ & $\Lambda^{*}$ & $1944 / 1975$ & $1976 / 2007$ & $\Lambda^{*}$ \\
\hline Mês & $\sum \mathrm{L}(\alpha, \beta ; \mathrm{xi})$ & $\sum \mathrm{L}(\alpha, \beta ; \mathrm{xi})$ & & $\sum \mathrm{L}(\alpha, \beta ; \mathrm{xi})$ & $\sum \mathrm{L}(\alpha, \beta ; \mathrm{xi})$ & \\
\hline Jan & $-197,8$ & $-201,9$ & 1,41 & $-187,0$ & $-192,2$ & 1,27 \\
\hline Fev & $-186,0$ & $-199,7$ & $10,30^{*}$ & $-190,5$ & $-190,8$ & 3,45 \\
\hline Mar & $-187,4$ & $-190,1$ & 3,64 & $-179,2$ & $-184,6$ & $6,36^{*}$ \\
\hline Abr & $-162,9$ & $-176,0$ & 2,11 & $-158,5$ & $-167,7$ & 3,29 \\
\hline Maio & $-155,2$ & $-172,1$ & 4,29 & $-154,1$ & $-170,6$ & $8,42^{*}$ \\
\hline Jun & $-134,6$ & $-120,9$ & 0,75 & $-153,1$ & $-144,1$ & 0,07 \\
\hline Jul & $-103,8$ & $-124,2$ & 0,73 & $-128,6$ & $-143,9$ & 0,66 \\
\hline Ago & $-108,5$ & $-105,6$ & 1,22 & $-127,6$ & $-133,7$ & 0,08 \\
\hline Set & $-144,0$ & $-169,3$ & $8,59^{*}$ & $-157,6$ & $-166,1$ & $9,84^{*}$ \\
\hline Out & $-177,9$ & $-186,1$ & 3,57 & $-170,1$ & $-182,9$ & $7,83^{*}$ \\
\hline Nov & $-188,6$ & $-185,5$ & 3,15 & $-183,0$ & $-175,8272$ & 2,73 \\
\hline \multirow[t]{2}{*}{ Dez } & $-202,5$ & $-194,5$ & $8,62^{*}$ & $-191,1$ & $-180,0$ & $15,54^{*}$ \\
\hline & \multicolumn{3}{|c|}{ Pindorama } & \multicolumn{3}{|c|}{ Ribeirão Preto } \\
\hline & $1952 / 1979$ & $1980 / 2007$ & $\Lambda^{*}$ & $1938 / 1972$ & $1973 / 2007$ & $\Lambda^{*}$ \\
\hline Mês & $\sum \mathrm{L}(\alpha, \beta ; \mathrm{xi})$ & $\sum \mathrm{L}(\alpha, \beta ; \mathrm{xi})$ & & $\sum \mathrm{L}(\alpha, \beta ; \mathrm{xi})$ & $\sum \mathrm{L}(\alpha, \beta ; \mathrm{xi})$ & \\
\hline Jan & $-161,8$ & $-170,6$ & $9,02 *$ & $-210,6$ & $-212,8$ & 0,16 \\
\hline $\mathrm{Fev}$ & $-166,2$ & $-170,0$ & 0,28 & $-207,3$ & $-215,0$ & 2,89 \\
\hline Mar & $-157,0$ & $-160,6$ & 0,36 & $-203,3$ & $-199,6$ & 0,01 \\
\hline Abr & $-140,2$ & $-139,9$ & 1,49 & $-166,6$ & $-190,1$ & $7,72^{*}$ \\
\hline Maio & $-129,0$ & $-143,0$ & 2,82 & $-157,1$ & $-181,2$ & 1,61 \\
\hline Jun & $-108,2$ & $-105,5$ & 3,72 & $-127,7$ & $-127,0$ & 0,65 \\
\hline Jul & $-92,8$ & $-100,6$ & 0,81 & $-112,4$ & $-126,5$ & 0,12 \\
\hline Ago & $-106,3$ & $-92,0$ & 0,05 & $-105,4$ & $-105,5$ & 0,53 \\
\hline Set & $-136,9$ & $-133,8$ & 0,12 & $-154,6$ & $-179,6$ & 1,25 \\
\hline Out & $-146,4$ & $-153,9$ & $7,60 *$ & $-191,3$ & $-190,6$ & 1,35 \\
\hline Nov & $-152,6$ & $-155,1$ & 0,59 & $-196,1$ & $-200,6$ & 1,14 \\
\hline \multirow[t]{3}{*}{ Dez } & $-168,3$ & $-161,6$ & 1,51 & $-215,0$ & $-209,7$ & $5,64^{*}$ \\
\hline & & Ubatuba & & \multicolumn{3}{|r|}{ Ubatuba } \\
\hline & $1936 / 1971$ & $1972 / 2007$ & $\Lambda^{*}$ & & $1936 / 1971$ & $1972 / 2007$ \\
\hline Mês & $\sum \mathrm{L}(\alpha, \beta ; \mathrm{xi})$ & $\sum \mathrm{L}(\alpha, \beta ; \mathrm{xi})$ & & Mês & $\Sigma \mathrm{L}(\alpha, \beta ; \mathrm{xi})$ & $\sum \mathrm{L}(\alpha, \beta ; \mathrm{xi})$ \\
\hline Jan & $-240,3$ & $-229,4$ & 3,45 & Jul & $-198,5$ & $-192,3$ \\
\hline Fev & $-240,5$ & $-235,1$ & 4,14 & Ago & $-200,9$ & $-183,2$ \\
\hline Mar & $-232,6$ & $-231,2$ & 4,06 & Set & $-206,2$ & $-209,5$ \\
\hline Abr & $-219,0$ & $-220,7$ & 0,16 & Out & $-216,4$ & $-213,9$ \\
\hline Maio & $-197,3$ & $-204,2$ & 2,87 & Nov & $-224,8$ & $-216,4$ \\
\hline Jun & $-199,0$ & $-190,2$ & 1,73 & Dez & $-228,5$ & $-219,0$ \\
\hline
\end{tabular}



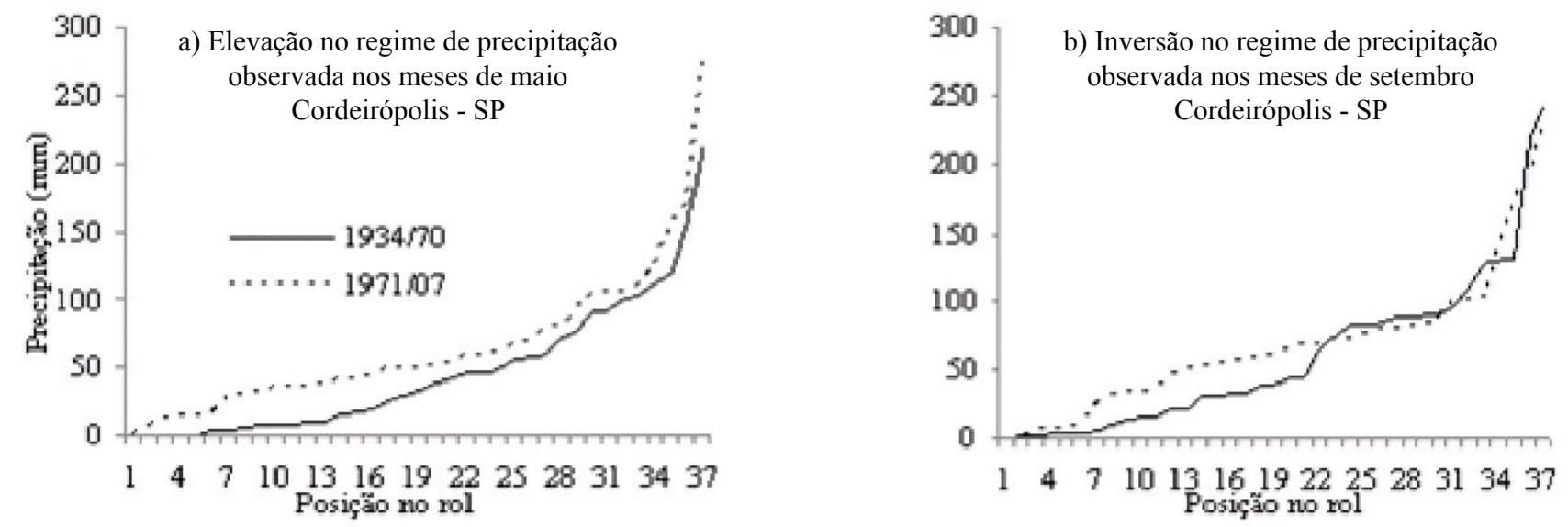

Figura 4 - Exemplo do termo elevação (a) e inversão (b) no regime de precipitação pluvial mensal da localidade de Cordeirópolis no Estado de São Paulo.

conforme é ilustrado na Figura 4a a para a região de Cordeirópolis.

Foi observada queda no regime mensal de precipitação apenas no mês de agosto para a localidade de Ubatuba. Nas demais séries mensais (em que foram observadas diferenças em todas as posições do rol de cada amostra) observou-se elevação no regime de precipitação pluvial. Nos demais meses (em que foram detectados $\Lambda^{*}$ significativos) há inversões das tendências de redução e aumento no regime de precipitação ao longo das amostras. Como exemplo, pode-se citar as amostras relativas ao mês de setembro na localidade de Cordeirópolis. Até a posição 23 do rol (o qual possui 37 posições) a amostra relativa aos anos de 1971 a 2007 apresentou um regime de precipitação superior aos observados no período entre 1934 a 1970. Entre as posições 24 a 33 observa-se inversão em tal característica. Nas posições 34 a 36 a amostra relativa aos anos de 1971 a 2007 volta a apresentor totais de precipitação superiores aos observados na de 1934 a 1970 e na última posição do rol há novamente inversão dessa característica. Dessa forma, nesses casos, apesar das probabilidades acumuladas de ocorrência, associadas à cada valor de precipitação, diferirem significativamente entre as duas amostras de cada agrupamento mensal (detecção de alterações no regime de precipitação), torna-se bastante difícil, determinar, por meio do $\Lambda^{*}$, se houve diminuição ou aumento no regime de precipitação pluvial. Com isso, indicações por parte de modelos meteorológicos que projetam cenários futuros de precipitação (inferindo, por exemplo, $15 \%$ de queda ou aumento na precipitação), bem como, a aplicação dessas indicações em possíveis alterações no zoneamento agrícola do estado de São Paulo deve-se considerar a possibilidade de que tais alterações possam ocorrer de forma distinta entre os diferentes percentis de uma distribuição mensal de precipitação. É também ilustrado na Figura 4b, a inversão no rol dos dados da localidade Cordeirópólis-SP para o mês de setembro. Na Tabela 3 são ilustradas as estatísticas u(t) relativas à séries mensais do estudo.

A aplicação do teste de MK não detectou, ao nível de $5 \%$ de significância, tendências (tanto de redução quanto de aumento) em nenhuma das 96 séries mensais de precipitação do estudo. Os valores da estatística $u(t)$ permaneceram sempre dentro do limite de confiança $[1,96 ;+1,96]$. É, entretanto, importante ressaltar que à exceção da localidade de Ubatuba, observa-se tendência não significativa (a 5\%) de elevação nos totais mensais de precipitação relativos ao mês de maio em todas as localidades analisadas. Em contrapartida, comparando-se os resultados das análises de ondeletas e do teste $\Lambda^{*}$, tornase evidente que as variações aleatórias, observadas dentro de cada série mensal, limitam a acurácia do teste MK na detecção de tendências climáticas no regime de precipitação pluvial das oito localidades. Nesse sentido, Frei e Schar (2000) afirmam que variações estocásticas exercem papel limitante na capacidade/probabilidade de detecção de tendências em séries meteorológicas finitas. A aplicação do MK nas 8 séries agrupadas e organizadas de forma análoga a que foi realizada para a análise de ondeletas (subtração das medianas mensais), também não indicou tendências climáticas significativas.

\section{CONCLUSÃO}

A principal característica das séries mensais de precipitação pluvial das oito localidades analisadas, é a elevada variabilidade temporal de seus valores. Nesse sentido, o tratamento de tais séries como sendo estritamente estacionárias, ou a condução de análises apenas no domínio da freqüência acarretará em perda de importantes informações sobre as forçantes moduladoras desse processo estocástico. Apesar dessa característica, não houve marcantes indicações 
Tabela 3 - Estatística u(t) relativa ao teste Mann-Kendall nas localidades de Campinas (1890/2007), Cordeirópolis (1934/2007), Jundiaí (1942/2007), Mococa (1942/2007), Monte Alegre do Sul (1942/2007), Pindorama (1951/2007), Ribeirão Preto (1937/2007) e Ubatuba (1935/2007), pertencentes ao Estado de São Paulo.

Estatística u(t) - limite de confiânça $[-1,96 ;+1,96]$

\begin{tabular}{ccccccccccccc}
\hline Local $\backslash$ Meses & jan & fev & mar & abr & maio & jun & jul & ago & set & out & nov & dez \\
Campinas & 0,51 & $-0,88$ & $-0,18$ & 0,57 & 1,26 & $-0,87$ & 0,52 & 0,52 & $-0,84$ & 0,04 & $-0,94$ & $-0,26$ \\
Cordeirópolis & 0,85 & $-0,56$ & $-0,54$ & $-0,29$ & 1,70 & $-0,18$ & 1,21 & $-0,78$ & 0,25 & $-1,06$ & $-0,73$ & $-0,61$ \\
Jundiaí & 1,79 & $-0,74$ & 0,12 & 0,74 & 1,78 & $-0,81$ & 1,07 & 0,58 & 1,69 & 0,05 & 1,32 & 1,00 \\
Mococa & 1,59 & $-0,30$ & $-1,42$ & 1,02 & 1,84 & $-1,49$ & 0,76 & $-0,48$ & 1,67 & $-0,30$ & 0,34 & 1,48 \\
MteAlegre & 0,17 & $-0,85$ & $-0,95$ & 1,06 & 1,92 & $-1,04$ & 0,76 & $-0,08$ & 1,79 & 0,82 & 0,68 & 1,15 \\
Pindorama & 1,86 & $-0,55$ & 0,12 & 1,24 & 1,40 & $-1,82$ & 1,06 & $-1,38$ & 0,87 & $-0,70$ & 0,81 & $-0,01$ \\
Rib.Preto & 0,34 & 0,60 & $-0,56$ & 0,17 & 1,78 & $-0,48$ & 0,83 & $-0,77$ & 1,67 & $-1,08$ & 0,85 & $-0,08$ \\
Ubatuba & $-1,10$ & $-1,43$ & $-1,33$ & $-0,54$ & $-0,52$ & $-0,63$ & $-0,10$ & $-1,55$ & 0,70 & 0,30 & $-0,42$ & $-1,78$ \\
\hline
\end{tabular}

de tendências de ordem climática no regime de totais mensais precipitação pluvial das localidades do estudo. A união dos resultados dos métodos utilizados indica, que as alterações detectadas estão fundamentalmente relacionadas às variações aleatórias do processo. Entretanto, especialmente para o mês de maio (à exceção da localidade de Ubatuba-SP), nota-se uma tendência pouco significativa de elevação nos totais mensais de precipitação pluvial.

A elevada variabilidade observada dentro de cada série mensal, também pode ser notada quando são comparadas (em um período homogêneo) as distribuições de distintos meses. Em uma mesma região, verificam-se distribuições com forma bastante próxima à gaussiana, caracterizando um período chuvoso, e distribuições fortemente assimétricas com forma exponencial "j”" invertido, caracterizando o período seco.

Sob o ponto de vista agrometeorológico, essas características, acima citadas, imprimem mesmo em um zoneamento probabilístico bem conduzido, considerável grau de incerteza na determinação do potencial de atendimento hídrico às culturas. Tal incerteza deve ser considerada na determinação de áreas aptas, inaptas e marginais.

\section{REFERÊNCIAS BIBLIOGRÁFICAS}

ALEXANDER, L.V.; ZHANG, X.; PETERSON, T.C.; CAESAR, J.; GLEASON, B.; TANK, A.M.G; HAYLOCK, M.; COLLINS, D.; TREVIN, B.; RAHIMZADEH, F.; TAGIPOU, A.; RUPA KUMAR, K.; REVADEKAR, J.; GRIFFITHS, G.; VINCENT, L.; STEPHENSON, D.; BURN, J.; AGUILLAR, E.; TAYLOR, M.; NEW, M.; ZHAI, P.; RUSTICUCCI, M.; VASQUEZ-AGUIRRE, J.L. Global observed changes in daily climate extremes of temperature and precipitation. Journal of Geophysical Research, Washington, v. 111, 2006. Disponível em: <http://www.agu.org/journals/ jd/jd0605/2005JD006290/>. Acesso em: 24 nov. 2008.
BACK, A.J. Aplicação de análise estatística para identificação de tendências climáticas. Pesquisa Agropecuária Brasileira, Brasília, v. 36, p. 717-726, 2001.

BLAIN, G.C.; KAYANO, M.T.; SENTELHAS, P.C.; LULU, J. Variabilidade amostral das séries mensais de precipitação pluvial na região de Campinas, São Paulo, Brasil (1890 a 2006) In: CONGRESSO BRASILEIRO DE METEOROLOGIA, 15., 2008, São Paulo. Anais... São Paulo: Sociedade Brasileira de Meteorologia, 2008. CD-ROM.

BLAIN, G.C. Avaliação e adaptação do Índice de Severidade de Seca de Palmer (PDSI) e do Índice Padronizado de Precipitação (SPI) às condições climáticas do Estado de São Paulo. 2005. 120p. Dissertação (Mestrado em Agricultura Tropical e Sub-tropical) - Instituto Agronômico, Campinas, 2005.

BLAIN, G.C.; PIEDADE, S.M.S.; CAMARGO, M.B.P.; GIAROLLA, A. Distribuição temporal da precipitação pluvial mensal observada no posto meteorológico do Instituto Agronômico em Campinas, SP. Bragantia, Campinas, v. 66, n. 2, p. 347-355, 2006.

BEN-GAI, T.; BITTAN, A.; MANES, A.; ALPERT, P.; RUBIN, $S$. Spatial and temporal changes in rainfall frequency distribution patterns in Israel. Theoretical and Applied Climatology, Wien, v. 61, p. 177-190, 1998.

FILHO, T.K.; ASSAD, E.D.; LIMA, P.R.S.R. Regiões pluviometricamente homogêneas no Brasil. Revista Brasileira de Pesquisa Agropecuária, Brasília, v. 40, n. 4, p. 311-322, 2005.

FREI, C.; SCHAR, C. Detection probability of trends in rare events: Theory and application to heavy precipitation in the Alpine region. Journal of Climate, Zurich, v. 14, p. 1568-1584, 2000.

GOOSSENS, C.; BERGER, A. Annual and seasonal climatic variations over the northern hemisphere and Europe during the lastcentury.Annales Geophysicae, Berlin, v.4,p. 385-400, 1986. 
GÖPFERT, H.; ROSSETTI, L.A.; SOUZA, J. Eventos generalizados e seguridade agrícola. Brasília: IPEA, 1993.65 p. HAYLOCK, M.R.;PETERSON, T.C.;ALVES,L.M.;AMBRIZZI, T.; ANUNCIAÇÃO, Y.M.T.; BAEZ, J.; BARROS, V.R.; BERLATO, M.A.; BIDEGAIN, M.; CORONEL, G.; CORRADI, V.; GARCIA, V.J.; GRIMM, A.M.; KAROLY, D.; MARENGO, J.A.; MARINO, M.B.; MONCUNILL, D.F.; NECHET, D.; QUINTANA, J.; REBELLO, E.; RUSTICUCCI, M.; SANTOS, J.L.; TREBEJO, I.; VINCENT L.A. Trends in total and extreme South American rainfall in 1960-2000 and links with sea surface temperature. Journal of Climate, Boston, v. 19, p. 1490-1512, 2006.

KAYANO, M.T.; BLAIN, G.C. Análisis de ondeletas de series mensuales de precipitación, temperaturas máximas y mínimas mensuales en Campinas. In: CONGRESO VENEZOLANO DE AGROMETEOROLOGIA, 4., 2007, Maracay. Anais... Maracay: Fundacite Táchira, 2007.

KELLER, T. Redução do risco climático na agricultura: uma abordagem probabilística: 1-Metodologia, principais aspectos. In: SIMPÓSIO INTERNACIONAL DE SECURIDADE E ZONEAMENTO AGRÍCOLA DO MERCOSUL, 1., 1998, Brasília. Anais... Brasília: Ministério da Agricultura e Abastecimento, 1998. p. 40-47.

KENDALL, M.A.; STUART, A. The advanced theory of statistics. Londres: Charles Griffin \& Company Limited, 1967. 690p.

MARENGO, J.; NOBRE, C.; RAIGOZA, D.; VALVERDE, M.; PISNITCHENKO, I.A.; OLIVEIRA, J.C.M. Boletim do Projeto: uso de cenários de mudanças climáticas regionais em estudos de vulnerabilidade e adaptação no Brasil e na América do Sul (GOF-UK-CPTEC), 2007. Disponível em: <www. cptec.inpe.br/mudancas_climaticas $>$. Acesso em: 1 nov. 2008. MORAES, J.M.; PELLEGRINO, G.; BALLESTER, M.V.; MARTINELLI,L.A.; VICTORIA, R.L. Estudo preliminar da evolução temporal dos componentes do ciclo hidrológico da bacia do Rio Piracicaba. In: SIMPÓSIO BRASILEIRO DE RECURSOS HÍDRICOS, 11.; SIMPÓSIODEHIDRÁULICA
E RECURSOS HÍDRICOS DOS PAÍSES DE LÍNGUA OFICIAL PORTUGUESA, 2., 1995, Recife. Anais... Recife: Associação Brasileira de Recursos Hídricos, 1995. p. 27-32.

NAKKEN, M. Wavelet analysis of rainfall-runoff variability isolating climatic from anthropogenic patterns. Environmental Modelling \& Software, v. 14, p. 283-295, 1998.

SCHUBNELL, P.R. Redução do risco climático na agricultura: uma abordagem probabilística. 2- Resultados obtidos. In: SIMPÓSIO INTERNACIONAL DE SECURIDADE E ZONEAMENTO AGRÍCOLA DO MERCUSUL, 1., 1998, Brasília. Anais... Brasília: Ministério da Agricultura e do Abastecimento, 1998. p. 48-57.

SCHICKEDANZ, P.T.; KRAUSE, G.F.; A test for the scale parameters of two gamma distributions using the generalized likelihood ratio. Journal of applied meteorology, Boston, v. 9, p. 13-16, 1969.

TORRENCE, C.; COMPO, G.P. A Practical Guide to Wavelet Analysis. Bulletin of the American Meteorological Society, Boston, v. 79, p. 61-78, 1998.

VINCENT, L.A.; PETERSON, T.C.; BARROS, V.R.; MARINO, M.B.; RUSTICUCCI, M.; CARRASCO, G.; RAMIREZ, E.; ALVES, L.M.; AMBRIZZI, T.; BERLATO, M.A.; GRIMM, A.M.; MARENGO, J.A.; MOLION. L.; MONCUNILL, D.F.; REBELLO, E.; ANUNCIAÇÃO, Y.M.T.; QUINTANA, J.; SANTOS, J.L.; BAEZ, J.; CORONEL, G.; GARCIA, J.; TREBEJO, I.; BIDEGAIN, M.; HAYLOCK, M.R.; KAROLY, D. Observed trends in indices of daily temperature extremes in South America 1960-2000. Journal of Climate, Zurich, v. 18, p. 5011-5023, 2005.

WILHITE, D.A. Drought as a natural hazard: Conceptions and definitions. In: Drought: A global assessment. Nebraska: Routledge, 2000. p. 111-120.

WILKS, D.S. Theoretical probability distributions. In: Statistical methods in the atmospheric sciences. San Diego: Academic Press, 2006. p. 102-104. 